\title{
Time dependent effect of chronic embryonic exposure to ethanol on zebrafish: morphology, biochemical and anxiety alterations
}

\begin{abstract}
Exposure to ethanol during critical period of development can cause severe impairments in the central nervous system (CNS). This study was conducted to assess the neurotoxic effects of chronic embryonic exposure to ethanol in the zebrafish, taking into consideration the time dependent effect. Two types of exposure regimen were applied in this study. Withdrawal exposure group received daily exposure starting from gastrulation until hatching, while continuous exposure group received daily exposure from gastrulation until behavioural assessment at $6 \mathrm{dpf}$ (days post fertilization). Chronic embryonic exposure to ethanol decreased spontaneous tail coiling at $24 \mathrm{hpf}$ (hour post fertilization), heart rate at $48 \mathrm{hpf}$ and increased mortality rate at $72 \mathrm{hpf}$. The number of apoptotic cells in the embryos treated with ethanol was significantly increased as compared to the control. We also measured the morphological abnormalities and the most prominent effects can be observed in the treated embryos exposed to $1.50 \%$ and $2.00 \%$. The treated embryos showed shorter body length, larger egg yolk, smaller eye diameter and heart edema as compared to the control. Larvae received $0.75 \%$ continuous ethanol exposure exhibited decreased swimming activity and increased anxiety related behavior, while withdrawal ethanol exposure showed increased swimming activity and decreased anxiety related behavior as compared to the respective control. Biochemical analysis exhibited that ethanol exposure for both exposure regimens altered proteins, lipids, carbohydrates and nucleic acids of the zebrafish larvae. Our results indicated that time dependent effect of ethanol exposure during development could target the biochemical processes thus leading to induction of apoptosis and neurobehavioral deficits in the zebrafish larvae. Thus it raised our concern about the safe limit of alcohol consumption for pregnant mother especially during critical periods of vulnerability for developing nervous system.
\end{abstract}

Keyword: Anxiety; Continuous exposure; Ethanol; Withdrawal exposure; Zebrafish 